\title{
Study in the Solid State, Framework Energy and Crystal Structure by X-Ray Diffraction Techniques of Cetirizine and Levocetirizine Dihydrochloride Used as an Antihistamines
}

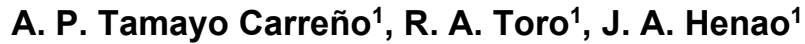 \\ ${ }^{1}$ Universidad Industrial de Santander, Carrera 27 con Calle 9, Bucaramanga, Colombia \\ angie.tamayo@correo.uis.edu.co
}

Cetirizine dihydrochloride and levocetirizine are antihistamines of second-generation that block histamine receptors $\mathrm{H}_{1}$, are widely used to treat allergic symptoms. These compounds belong to the class of antihistamines piperazine type and like other secondgeneration antihistamines, are considered non-sedating [1]. The crystal structure of cetirizine dihydrochloride has been solved and refined using X-ray powder diffraction data and optimized using Density Functional Theory (DFT) techniques. The cetirizine dihydrochloride Fig. 1, crystallized in a monoclinic system and space group P2 $1 / \mathrm{n}\left(\mathrm{N}^{\circ}\right.$ 14) with parameters $a=13,6663(3) \AA$, $b=7,0978$ (7) $\AA, c=23,8311(1) \AA, \beta=102,488(3)^{\circ}, V=2251,06 \AA^{3}$ and $Z=4$. On the other hand, the levocetirizine dihydrochloride Fig. 1 , crystallized in a monoclinic system and space group P2 1 ( $\left.\mathrm{N}^{\circ} 4\right)$ with parameters $a=13,5450(7) \AA, b=7,0719(9) \AA, c=24,0527(2) \AA$, $\beta=98,159(3)^{\circ}, V=2280,65 \AA^{3}$ and $Z=2$. In both crystalline structures there are multiple hydrogen bonds intra and inter molecular, $\pi$ interactions and hydrogen- $\pi$ interactions. The molecular packing and crystal energy are dominated by Van der Waals attractions according to Hirshfeld surfaces. Finally, the crystal structure was optimized with DFT and all non-H bond distances and angles were subjected to restraints, based on a Mercury Mogul Geometry Check of each molecule.

A search in the Cambridge Structural Database (CSD) [2] confirmed the absence of reports for the crystal structure of cetirizine dihydrochloride and levocetirizine. However, there are several reports of cetirizine dihydrochloride and levocetirizine in the $P D F$ 4/Organics database [3] contains four entries PDF 00-058-1973, 00-058-1974 and 00-058-1975, corresponding to unindexed patterns about cetirizine dihydrochloride, dextrocetirizine dihydrochloride and levocetirizine dihydrochloride, respectively; PDF 00-066-1627 corresponding an experimental pattern for cetirizine dihydrochloride, according to this report, it crystallizes in a monoclinic cell with parameters $a=24.1256(7) \AA, b=7.07588(7) \AA, c=13.5196(4) \AA \beta=98.0028(28)^{\circ}$ and $V=2285.45 \AA^{3}$ in space group P2 ${ }_{1} / \mathrm{n}\left(\mathrm{N}^{\circ} 14\right)$.
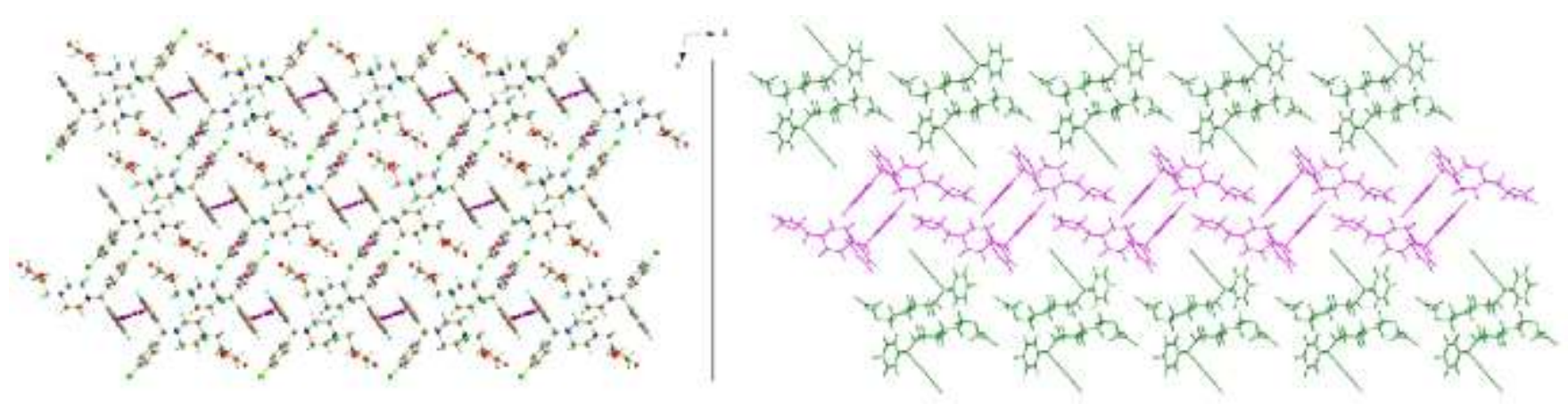

Figure 1. Cetirizine dihydrochloride crystal structure show $\pi$-interactions (left); Levocetirizine dihydrochloride crystal structure with two independent crystallographic molecules (right).

[1] Pflum, D. A., Krishnamurthy, D., Han, Z., Wald, S. A. \& Senanayake, C. H. (2002). Tetrahedron Lett. $43,923$.

[2] Groom, C. R., Bruno, I. J., Lightfoot, M. P. \& Ward, S.C. (2016) The Cambridge structural database, Acta Crystallogr. B72, $171-179$.

[3] ICDD, PDF-4/Organics 2020 (database), edited by S. Kabekkodu, International Centre for Diffraction Data, Newtown Square, PA, USA.

Keywords: $\mathbf{X}$ ray diffraction; Rietveld refinement; simulated annealing; DFT.

Acknowledgements: The authors thanks to Laboratorio de Rayos X UIS-PTG of the Universidad Industrial de Santander (UIS) Bucaramanga, Colombia and the Vicerrectoria de Investigación y Extensión, for the recording of diffraction data. 